\title{
Panorama das Pesquisas sobre Crenças Coletivas de Eficácia Docente
}

\author{
Maély Holanda Ramos ${ }^{1}$, Simone Souza Silva ${ }^{1}$, Fernando Ramos Pontes ${ }^{1}$ \\ 1 Universidade Federal do Pará, Brasil
}

\begin{abstract}
Resumo: Eficácia coletiva docente refere-se às crenças de um professor na capacidade dos membros do seu grupo para obterem sucesso numa determinada ação pedagógica. Este artigo tem o objetivo de construir um panorama das pesquisas sobre eficácia coletiva de professores, na perspectiva social cognitiva. Toma-se por base a Teoria Social Cognitiva de Albert Bandura. Para tanto, foram analisados 30 artigos resultantes de buscas realizadas nas bases de dados da CAPES-periódicos, referentes ao período entre 2000 a 2014. Para análise dos dados aplicou-se estudo das redes de relações para identificar as conexões entre as variáveis investigadas nos artigos por meio de grafos. Os resultados indicaram que nos artigos investigados algumas variáveis alcançaram destaque, pois foram mais frequentemente relacionadas à eficácia coletiva, a saber: autoeficácia docente, desempenho de alunos e situação socioeconômica. Isto indica que estas variáveis podem influenciar a eficácia coletiva. Notou-se que os estudos indicaram uma influência recíproca entre autoeficácia e eficácia coletiva. Isto sugere que, apesar de serem construtos distintos, estas variáveis são interdependentes.
\end{abstract}

Palavras-chave: Eficácia coletiva; Professores; Revisão Sistemática.

Overview of Research on Teacher's Collective Efficacy Beliefs: Teacher collective efficacy refers to the beliefs of a teacher regarding the capacity of group members to succeed in a particular pedagogical action. This article aims to make an overview of research on collective efficacy of teachers in a social cognitive perspective framed by the Social Cognitive Theory of Albert Bandura. A total of 30 articles were analyzed resulting from searches in the databases of the CAPES-Journals from 2000 to 2014. For data analysis, we applied the study of networks relations to identify the connections among the variables investigated on the articles through graphs. Results indicate that in those papers some variables were most often related to collective efficacy, namely: teacher's self-efficacy, student performance and socioeconomic status. This indicates that these variables can influence collective efficacy. It was observed that the studies indicate a reciprocal influence between self-efficacy and collective efficacy. This suggests that, even if different constructs, these variables are interdependent.

Keywords: Collective efficacy; Teachers; Systematic Review.

Todo o indivíduo tem capacidade para selecionar, criar e transformar as diversas circunstâncias ambientais às quais é exposto. Essa capacidade permite influenciar cursos de acontecimentos, motivar, orientar e reorientar as suas ações (Bandura, 1989, 2001).

Bandura (1986) explica o funcionamento humano a partir de processos cognitivos, os quais são autorregulados e autoreflexivos, gerando adaptações e mudanças. Esses processos cognitivos são atividades cerebrais emergentes que exercem uma influência determinante no comportamento das pessoas. Isso porque a mente humana é geradora, criativa e reflexiva, não é apenas reativa. Nessa concepção, as pessoas não são dirigidas somente pelas circunstâncias ambientais ou por impulsos internos camuflados. Os indivíduos são agentes capazes de fazer coisas acontecerem de forma proativa, podendo exercer controle sobre as próprias vidas, estabelecendo limites para o autodirecionamento.

É importante destacar que as crenças que o indivíduo desenvolve sobre si mesmo e, mais especificamente, sobre sua capacidade de controlar seu próprio comportamento, são fundamentais, pois interferem na tomada de decisão (Bandura, 2008). As crenças são fatores que funcionam como mecanismos de mudança de comportamento, influenciando-o por meio da expectativa de sucesso (Bandura, 1997).

Na perspectiva da Teoria Social Cognitiva criada por Bandura (1986), a crença do indivíduo em si mesmo é definida como autoeficácia, isto é, as percepções ou expectativas das pessoas sobre si mesmas, relacionadas às tarefas desempenhadas, nível de esforço, persistência, nível de estresse, etc. Para Bandura

${ }^{1}$ Dados de contacto para correspondência: Maély F. H. Ramos; Rua Augusto Corrêa, N o 1, Instituto de Ciências da Educação Campus Profissional; CEP: 66075-110, Brasil.E-mail: maelyramos@hotmail.com. 
(1997), as crenças de autoeficácia são julgamentos que as pessoas desenvolvem sobre suas próprias capacidades de organizar e executar ações.

A percepção de eficácia coletiva, por sua vez, refere-se às crenças de um indivíduo na capacidade dos membros do seu grupo para obterem sucesso numa determinada ação e, quando compartilhadas, exercem influência sobre o comportamento de seus integrantes. A dimensão de grupo, que caracteriza o constructo de eficácia coletiva, procura explicar o comportamento a partir da capacidade de gerenciar ações e tarefas ao longo da vida. Assim, a eficácia coletiva, quando é compartilhada, pode potencializar capacidades conjuntas para organizar e executar cursos de ações requeridos para se produzirem determinados níveis de tarefas, bem como para superar falhas cometidas (Bandura, 1986, 1997).

A eficácia coletiva pode ser aplicada a diferentes ambientes institucionais. Em relação às crenças do grupo, o seu aspecto organizacional diz respeito às capacidades de desempenho de um sistema social como um todo. Tal compreensão estende-se a empresas, grupos políticos, associações esportivas, instituições escolares, entre outros. Especificamente no campo escolar, a eficácia coletiva deve ser analisada e compreendida respeitando as características próprias do ambiente de ensino e das relações que se desenvolvem (Bandura, 2008).

\section{Eficácia Coletiva de Professores}

Eficácia coletiva docente refere-se aos julgamentos dos professores de uma escola sobre a capacidade do conjunto de educadores de organizar e executar cursos de ação exigidos para se conseguirem resultados positivos junto aos alunos (Goddard, Hoy, \& Hoy, 2004). Desse modo, eficácia coletiva de professores refere-se à percepção docente quanto à capacidade que o grupo de professores tem de ajudar os seus alunos a aprender (Bandura, 1993). Não por acaso, resultados de pesquisas indicam relação significativa entre eficácia coletiva de professores e resultados acadêmicos dos alunos (Bandura, 1993; Goddard, 2001, 2002; Klassen, Tze, Betts, \& Gordon, 2011; McCoach \& Colbert, 2010).

Considerada como importante aspecto do contexto escolar (Bandura, 1993, 1997), a eficácia coletiva pode explicar, em parte, os efeitos da ação docente sobre o sucesso acadêmico dos estudantes, de modo a contribuir para a compreensão de como as escolas apresentam resultados diferentes entre si (Goddard, Hoy, \& Hoy, 2000). Vale destacar que não se pode perder de vista fatores que devem ser considerados nesse contexto, como os aspectos sociodemográficos (Goddard, 2001), socioeconômicos (Parker, Hanna \& Topping, 2006; Tchannen-Moran \& Barr, 2004), bem como aqueles relacionados com infraestrutura, suporte pedagógico, questões sociais e políticas de cada escola (Adams \& Forsyth, 2006; Skaalvik \& Skaalvik, 2007).

Professores são membros de organizações escolares e as suas crenças partilhadas influenciam o ambiente social no qual estão inseridos. Assim, o contexto escolar é propício para se compreenderem os efeitos das crenças de eficácia coletiva, uma vez que seus membros atuam de forma interdependente, visando objetivos em comum para o grupo, bem como metas individuais (Bandura, 1993). Nesse espaço, são vivenciadas tanto dificuldades em comum, quanto sucessos alcançados, determinando o nível de crenças acerca da capacidade de desempenhar ações em prol da instituição, e, também, atuando como produto da interação dinâmica dos membros do grupo.

Diferentes estudos constatam a influência exercida pelas crenças de eficácia coletiva de professores em vários aspectos do contexto escolar. Professores que acreditam na capacidade coletiva para ensinar os seus alunos de forma eficaz, contribuindo para o desenvolvimento da sua escola (Goddard, LoGerfo, \& Hoy, 2004), sentem-se encorajados a partilhar decisões organizacionais (Ross, Hogaboam-Gray, \& Gray, 2003), e, consequentemente, exercem algum tipo de ação sobre as normas sociais do espaço escolar (Goddard, 2001). Níveis elevados de eficácia coletiva favorecem escolas com professores mais capazes de lidar com alunos que apresentam dificuldades de aprendizagem, de apoiar os pais e de tê-los como parceiros no processo de ensino (Bandura, 1997).

Considerando a importância da eficácia coletiva docente no ambiente escolar, entende-se que é fundamental compreender que fatores influenciam essas crenças e como eles se relacionam. Desta forma, acredita-se que a análise das crenças de eficácia coletiva sob a ótica de técnicas de análise com grafos pode contribuir para o aprofundamento do conhecimento acerca destas relações. Através de grafos, é possível identificar as interações entre diferentes elementos por meio de métricas e representações geométricas, caracterizando os relacionamentos, numa perspectiva global e integrativa (Szwarcfiter, 1984).

Diante do contexto apresentado, o presente estudo tem como proposta construir o panorama dos estudos sobre eficácia coletiva docente na perspectiva social cognitiva, a partir da análise de artigos publicados no período de 2000 a 2014, no banco de dados da Capes-periódicos, com técnicas de análise com grafos. 


\section{MÉTODO}

Esta pesquisa apresenta uma perspectiva descritiva e exploratória sobre os trabalhos desenvolvidos acerca da eficácia coletiva docente nos últimos anos. Para tanto, foram desenvolvidas sete etapas baseadas nas orientações da Colaboração Cochrane para a realização de revisões sistemáticas, a saber: (1) formulação da pergunta/problema; (2) localização e seleção dos estudos; (3) avaliação crítica dos estudos; (4) coleta de dados nos artigos - variáveis a serem estudadas; (5) análise e apresentação dos dados; (6) interpretação dos dados; (7) aprimoramento e atualização da revisão (Cordeiro, Oliveira, Rentería, \& Guimarães, 2007).

A Colaboração Cochrane é uma organização internacional que visa contribuir para a tomada de decisões sobre intervenções no cuidado da saúde, por meio de revisões sistemáticas. Para tanto, emprega-se um criterioso método de seleção e avaliação de artigos para reunir as mais relevantes informações sobre uma determinada questão, buscando melhorar a qualidade das revisões sistemáticas. Apesar de ser direcionado a estudos de carácter clínico, de intervenção e de acurácia, o método da Colaboração Cochrane pode ser adaptado para atender revisões de outra natureza e em outras áreas do conhecimento, como é o caso da presente pesquisa (Biruel \& Pinto, 2011).

\section{O Percurso}

Inicialmente definiu-se a pergunta central desta pesquisa, a saber: Qual o panorama das pesquisas sobre eficácia coletiva docente? Sequencialmente realizou-se a $2^{\underline{a}}$ etapa (localização e seleção dos estudos) da revisão sistemática, que envolveu a definição de alguns descritores relacionados com a temática. Nesta seleção realizou-se a consulta aos Descritores em Ciências da Saúde (DECS), que é utilizado para identificar descritores numa linguagem acadêmica, na indexação de artigos científicos. Assim, instituiramse os seguintes descritores: collective efficacy, collective efficacy beliefs, teacher collective efficacy, collective perceptions, social cognitive theory, teacher efficacy, school.

Posteriormente, construiram-se estratégias de busca para serem submetidas às bases de dados, procedendo a buscas dos descritores nos títulos, resumos e palavras-chave dos artigos. Para tanto aplicaram-se os operadores booleanos (AND, OR) aos descritores selecionados. Desta maneira, formaram-se duas estratégias de busca em forma de equação (inglês e português), a saber:

- $\quad$ Equação 1

(collective efficacy OR collective efficacy beliefs OR teacher collective efficacy OR collective perceptions) AND school AND teacher AND (efficacy beliefs OR teacher efficacy) AND (theory social cognitive OR social cognitive theory).

\section{- $\quad$ Equação 2}

(eficácia coletiva OR crenças de eficácia coletiva OR crenças coletivas) AND (escola OR colégio) AND (professor OR docente) AND (Teoria Social Cognitiva OR perspectiva social cognitiva).

Para o delineamento da pesquisa, foram pré-determinados alguns critérios de inclusão, sendo esses: conter no título o termo collective efficacy ou eficácia coletiva; estar completo e disponível; artigos empíricos ou estudos de revisão sistemática; apenas artigos dos últimos 14 anos (2000 a 2014); artigos em inglês e português; ter sido revisado por pares; e ter como foco investigar o constructo da eficácia coletiva de professores, na perspectiva social cognitiva. Todos os achados que não obedeceram aos critérios de inclusão mencionados foram excluídos. Os artigos foram selecionados ( $2^{\mathrm{a}}$ etapa) na base de dados da CAPES-Periódicos, que é uma das maiores bibliotecas virtuais do mundo; resume conteúdo científico de alto nível, com publicações nacionais e internacionais e contém artigos e revistas de diferentes áreas do conhecimento, entre outras produções. Ressalta-se que este diretório possui importante abrangência quanto aos trabalhos publicados referentes ao objeto desta pesquisa, pois tem acesso a estudos de meta-análises e de revisões sistemáticas e inclui o SCOPUS na sua base de dados.

Após a seleção dos artigos, foram realizados dois testes de relevância (3a etapa) compostos de listas de perguntas objetivas para refinar o conteúdo dos artigos e possibilitar a avaliação de relevância. 0 Teste de Relevância I foi aplicado aos resumos e métodos dos artigos por apenas um avaliador e foi composto das seguintes questões com possibilidade de respostas negativas e positivas, a saber: o estudo está de acordo com o tema investigado? Foi publicado no período estipulado? É um estudo envolvendo professores? Foi publicado nos idiomas indicados na proposta da pesquisa? Tem acesso livre a pesquisadores (acesso ao texto completo)? Utiliza a Teoria Social Cognitiva como base de fundamentação?

No Teste de Relevância II foram considerados apenas os artigos aprovados no Teste de Relevância I e foram analisados para avaliação das informações publicadas. 0 teste II foi realizado de forma 
independente por dois juízes com conhecimento na área, estando composto das seguintes perguntas baseadas em Azevedo (2010): 0 objetivo do estudo tem relação com o que está sendo estudado? 0 método está descrito com clareza (referente à indicação de todas as etapas e da classificação do método; natureza da pesquisa; informações sobre população/amostra; instrumentos de coleta; procedimentos de coleta; verificação de informações importantes omitidas)? Os resultados merecem credibilidade (verificação dos procedimentos de análise e técnicas utilizadas para o alcance dos objetivos de estudo)? Por meio da análise dos artigos quanto às perguntas supracitadas, os juízes informaram se os estudos poderiam ser incluídos na presente revisão sistemática.

Após a aplicação do Teste de Relevância II, foi verificado o índice de confiabilidade entre os pesquisadores, a partir do seguinte cálculo:

$$
\mathrm{IC}=\mathrm{A} \times 100 / \mathrm{A}+\mathrm{D}
$$

Considera-se, neste caso, a legenda: IC = índice de concordância; $\mathrm{A}=$ concordância; $\mathrm{D}=$ discordância. Esta é uma técnica de triangulação de pesquisadores que tem como objetivo aumentar a probabilidade de que os resultados de um estudo sejam confiáveis e dignos de credibilidade. É aceitável IC $>80 \%$ (Pereira, 2006).

Calculou-se ainda o IC por meio da medida estatística de concordância Kappa de Cohen. Ressalta-se que esta medida varia de +1 a -1 , onde +1 significa que os juízes concordaram totalmente, 0 indica que não existe nenhuma relação entre a classificação dos dois juízes, e -1 representa que os avaliadores fizeram classificações exatamente opostas. Considera-se que: escores Kappa $>0,80$ indicam nível de concordância excelente; entre 0,60 e 0,80 - bom IC; entre 0,40 e 0,60 - IC regular; escore Kappa < 0,40 representa um índice de concordância ruim (Dancey \& Reidy, 2006).

Após a aplicação dos testes de relevância, iniciou-se a coleta de dados nos artigos (4⿳亠丷a etapa), com a delimitação das variáveis a serem investigadas. Escolheu-se focar a análise em apenas uma categoria de dados: os resultados dos artigos investigados. Sendo assim, para mineração e análise dos dados (5 ${ }^{\mathbf{a}}$ etapa), foram utilizados os seguintes programas: (1) EXCEL 2010 e SPSS 20 - para mineração e realização de uma estatística de tendência central que informa como a amostra é na sua totalidade, ou em média (Dancey \& Reidy, 2006); (2) MENDELEY - para organização das referências, bem como para o confronto das duplicidades, excluindo artigos repetidos e para o gerenciamento geral da base de dados; (3) NodeXL - na análise das redes de relações utilizando grafos, para explorar as conexões categóricas e estudo das variáveis mais investigadas nesta área de conhecimento nos últimos anos.

Um grafo consiste em conjuntos de vértices (elementos) e arestas (conexão entre os vértices). Com os caminhos de um grafo, forma-se uma estrutura topológica que caracteriza as relações entre os elementos, permitindo a visualização da complexidade das relações (Szwarcfiter, 1984).

Para análise das relações com grafos a partir do NodeXL foram calculadas as seguintes métricas: (1) Grau de Centralidade (in-degree/out-degree) - para quantificar o número de ligações associadas a um elemento; (2) Relevância do Vértice (PageRank) - para medir o grau de importância de um vértice (Szwarcfiter, 1984). Após a organização e análise dos dados, foi feito o processo de interpretação das informações, a partir da Teoria Social Cognitiva, e posterior construção e aprimoramento da revisão sistemática (6 ${ }^{\underline{a}}$ e $7 \stackrel{a}{a}$ etapas).

\section{RESULTADOS}

Foram encontrados, na base de dados da CAPES-Periódicos, 161 artigos a partir dos termos de busca prédeterminados e seus cruzamentos. No refinamento inicial, 27 artigos foram excluídos por estarem repetidos. Posteriormente, foi aplicado o Teste de Relevância I que resultou na exclusão de 101 objetos, pois não atenderam aos critérios de inclusão e não responderam positivamente a todas as questões do teste. Destaca-se que o grande número de exclusão se deve ao fato de que a maioria dos artigos não utilizou a Teoria Social Cognitiva como base de fundamentação (75\%). Restaram, portanto, 33 objetos, que representaram, apenas, $20 \%$ do total de artigos levantados inicialmente.

Sequencialmente, os artigos aprovados no Teste de Relevância I foram submetidos ao Teste de Relevância II e sofreram uma nova diminuição, totalizando, apenas, 30 objetos. No Teste de Relevância II, somente três artigos foram excluídos pois os métodos não estavam claros.

No teste de relevância II, calculou-se o Índice de Concordância (IC) que variou entre 97\% e 100\%. Na medida estatística de concordância Kappa de Cohen alcançou-se excelente escore $(k>0,80)$. Estes procedimentos foram realizados para garantir a consistência e a confiabilidade do trabalho de análise. Percebe-se que não houve grandes discordâncias entre os pesquisadores quanto à seleção dos artigos para a presente revisão sistemática. Nos poucos casos de discordância, os objetos em questões passaram por nova avaliação para definição da inclusão ou exclusão dos mesmos. 
Do total de objetos inicialmente levantados, 161 foram excluídos, alcançando 80\% dos artigos. É comum obter-se um grande percentual de exclusão em estudos de revisão sistemática (Pereira, 2007). Devem-se considerar os diferentes tipos de abordagens que dão ao constructo investigado uma variedade de perspectivas de análise, já que isto influencia a seleção dos objetos. No caso da temática investigada nesta revisão, percebe-se que a eficácia coletiva docente, na perspectiva social cognitiva, ainda tem sido pouco explorada, necessitando de maior atenção por parte dos pesquisadores desta área (Klassen, Usher, et al., 2010). Isto justifica o número limitado de artigos selecionados.

\section{Variáveis Investigadas nos Estudos}

Para o estudo das relações entre as variáveis indicadas nos resultados dos artigos investigados por meio de multigrafos, utilizou-se a ferramenta NodeXL. Os multigrafos foram organizados em dois grupos: (1) correlações positivas; (2) correlações negativas. Considera-se que as Figuras 1 e 2 são multigrafos, pois se notou a ocorrência de arestas duplas ou múltiplas entre um mesmo par de vértices (Szwarcfiter, 1984). Escolheu-se analisar as correlações entre as variáveis, pois foram as relações mais investigadas nos artigos selecionados para esta revisão sistemática. Por meio do delineamento correlacional (correlações positivas e negativas), é possível concluir a forma como as variáveis se associam (magnitude e direção). Com esta técnica estatística, observa-se como as variáveis mudam, sem, no entanto, estabelecer uma relação causal entre elas. Indica-se que quando valores numa variável mudam valores, em outra também mudam de forma previsível (Dancey \& Reidy, 2006). Optou-se por incluir neste estudo apenas as correlações significativas, sem delimitação de níveis de magnitudes.

Para melhor interpretar os multigrafos (Figuras 1 e 2) deve-se considerar que: (1) correlações positivas - cada vez que $\mathrm{x}$ aumenta, $\mathrm{y}$ aumenta um determinado valor; (2) correlações negativas - cada vez que $\mathrm{x}$ aumenta, $\mathrm{y}$ diminui um valor de forma também constante.

Enfatiza-se que cada aresta representa a ocorrência de uma determinada relação num artigo. Arestas duplas e/ou múltiplas entre um mesmo par de vértices dispõem-se de forma sobreposta. Deste modo, em termos de visualização, quanto mais larga for a aresta, isso indicará um maior número de referências de ligações entre vértices (variáveis) advindas de artigos diferentes. A quantidade de ligações é indicada por meio de métricas específicas (Degree) disponibilizadas pelo NodeXL. Ressalta-se que os vértices mais importantes foram destacados nos multigrafos (Figuras 1 e 2), de modo que quanto maior for o círculo representativo de cada vértice, mais importante é este elemento no multigrafo.

Utilizou-se como principal medida de centralidade a métrica PageRank. Esta medida indica que um vértice é mais conectado aos demais do grafo e, que por isso, é considerado importante. Esta medida considera também o nível de importância dos elementos com os quais um vértice se conecta. Se um vértice $\mathrm{x}$, se conecta a outros vértices ( $\mathrm{y}$ e z), que também são importantes no grafo ou multigrafo, os escores do PageRank serão mais elevados. Desta forma, pode-se definir o PageRank como o grau de importância do vértice $\mathrm{x}$, calculada pela soma da importância dos vértices que apontam para $\mathrm{x}$ (Matos, 2013; Pinheiro, 2013; Sousa, 2010). A Figura 1 apresenta o multigrafo das correlações positivas.

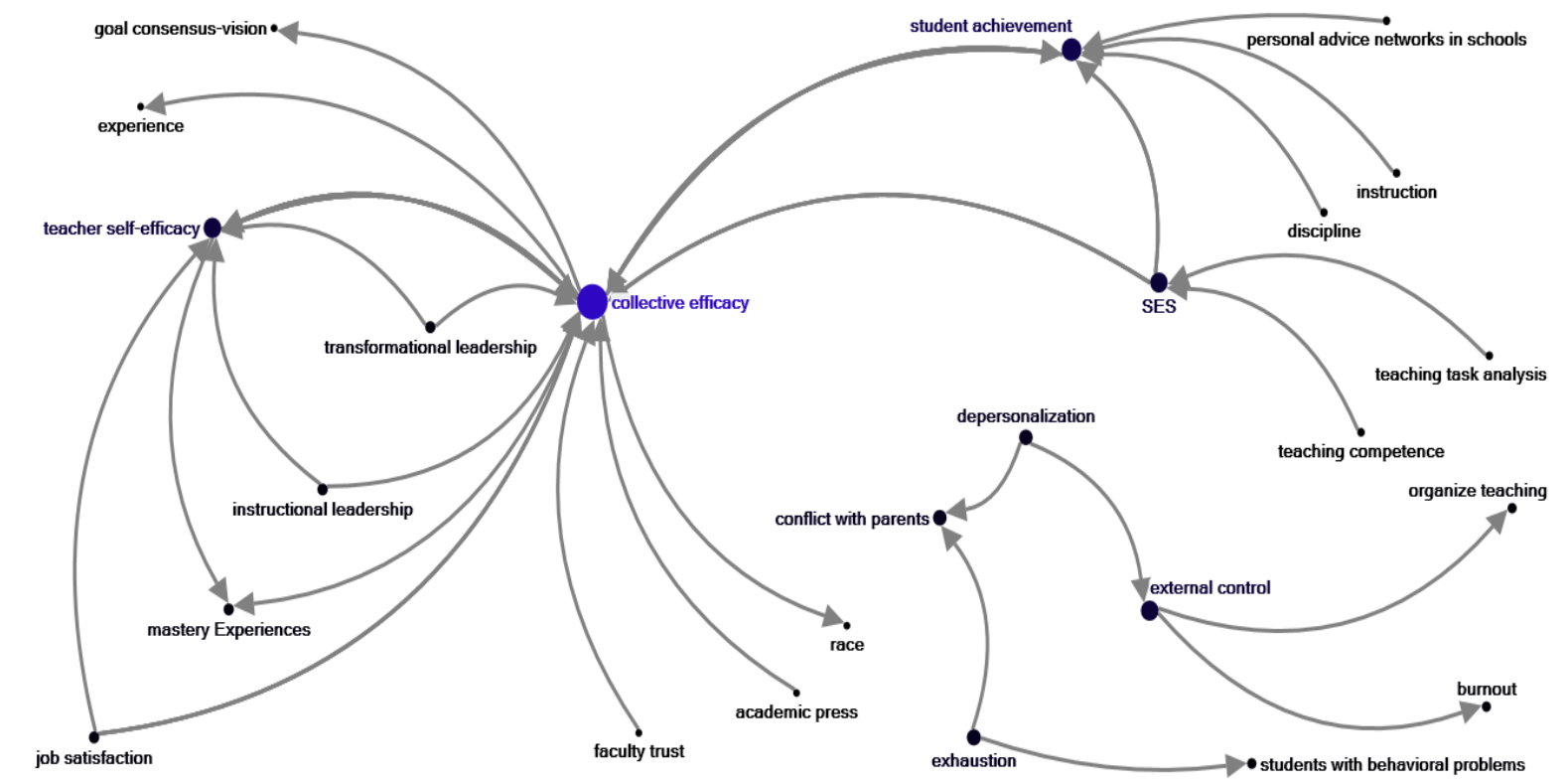

Figura 1. Multigrafo - Redes de correlações positivas. 
Variáveis - correlações positivas

A Figura 1 apresenta o multigrafo que sintetiza os resultados das correlações positivas extraídos dos artigos selecionados para esta revisão sistemática. Desta forma, foram destacados os elementos por ordem de centralidade, a saber: (1) eficácia coletiva; (2) desempenho de alunos; (3) autoeficácia; (4) situação socioeconômica - SES; (5) controle externo. Utilizou-se como ponto de corte escores na métrica PageRank $>$ 1,6. Considera-se que este ponto de corte indica melhor as variáveis mais importantes dos artigos.

O vértice mais importante foi a eficácia coletiva, com maior escore de relevância (PageRank) 4,6. Esta variável estabeleceu conexão com 12 outros elementos do multigrafo, tais como: consenso de metas, experiência, raça, capacidade de confiança dos docentes, entre outros. Ressalta-se, no entanto, que houve ligação mais forte com os vértices desempenho de alunos e autoeficácia.

0 vértice desempenho de alunos foi o segundo mais relevante elemento do multigrafo (Figura 1) com PageRank de 2,1, fazendo conexão com outros cinco vértices. Foi positivamente correlacionado aos seguintes elementos: instrução, disciplina, redes de aconselhamento pessoal na escola e situação socioeconômica (SES).

A variável desempenho de alunos formou, ainda, um par de vértices de múltiplas arestas (10), em dois sentidos (in-degree e out-degree) com a eficácia coletiva. Isto significa que 10 artigos investigaram esta relação, confirmando a forte ligação entre ambas. As pesquisas apontam que a eficácia coletiva docente pode estar relacionada ao desempenho dos alunos, no entanto, deve-se considerar que outros fatores do processo de ensino-aprendizagem que também podem estar associados à eficácia coletiva, tais como os aspectos socioeconômicos (Cybulski, Hoy, \& Sweetland, 2005; Goddard, 2001; Goddard, Logerfo, \& Hoy, 2004; Hoy, Sweetland, \& Smith, 2002; Moolenaar, Sleegers, \& Daly, 2012).

Goddard (2001) investigou a variabilidade da percepção dos professores em relação às crenças de eficácia coletiva docente, analisando também a relação entre esta variável e o desempenho dos alunos. Participaram da pesquisa 452 professores de 91 escolas do Ensino Fundamental nos Estados Unidos. Os resultados indicaram correlação positiva entre desempenho acadêmico e a eficácia coletiva $(r=.77$, $p<.001)$.

Hoy et al. (2002) buscaram construir um modelo teórico para explicar o desempenho dos alunos. Percebeu-se que a eficácia coletiva docente é uma variável-chave, e também teve correlação positiva com o desempenho dos alunos $(r=.65, p<.001)$. 0 estudo realizou-se em 97 escolas de ensino médio em Ohion, nos Estados Unidos. Entende-se que o forte senso de eficácia coletiva pode levar os professores a serem mais persistentes nos seus esforços para conseguir melhores resultados no processo de ensinoaprendizagem, superando possíveis dificuldades.

0 vértice autoeficácia foi o terceiro elemento mais importante do multigrafo, com PageRank de 1,7, estabelecendo conexão com cinco vértices. Houve correlação positiva entre as variáveis satisfação no trabalho, liderança instrucional, liderança transformacional e a autoeficácia, sendo que esta, por sua vez, se correlacionoupositivamente com experiências de maestria.

A autoeficácia também se conectou à eficácia coletiva, constituindo um par de vértices de múltiplas arestas (11). Estes dados indicam que 11 estudos investigaram esta relação nos quais foi identificada relação entre ambos os constructos.

Kurz e Knight (2004), nas suas investigações, constataram que a correlação entre eficácia coletiva e autoeficácia é positiva $(r=0.23, p<0.05)$, embora entendam que é razoável dizer que ambos os constructos são interdependentes. Esses resultados indicaram que forças distintas no ambiente de trabalho moldam a eficácia coletiva e a autoeficácia. Percepções diferentes dos professores a respeito das suas próprias crenças sobre a capacidade de gerar mudanças significativas e as suas crenças na capacidade dos seus colegas, podem contribuir para provocar uma diferença entre as crenças coletivas e pessoais de eficácia docente.

Skaalvik e Skaalvik (2007) examinaram a relação entre autoeficácia e eficácia coletiva em 244 professores do Ensino Fundamental e do Ensino Médio da Noruega. A análise fatorial realizada nos dados indicou que ambas variáveis são positiva e fortemente correlacionadas.

Os vértices satisfação no trabalho docente e eficácia coletiva, formaram um par de vértices com múltiplas arestas (3), apontando para uma forte conexão entre as variáveis, pois três artigos investigaram essa relação. Klassen, Tze, et al. (2010) defenderam que contextos culturais apontam, especificamente, diferenças significantes nos papeis desempenhados pela eficácia coletiva e as suas ligações com a satisfação no trabalho. Esse estudo é um dos primeiros a examinar as crenças coletivas de professores usando uma estrutura intercultural, investigando dados de países diferentes (dois países da América do Norte - Canadá e Estados Unidos, e um país do Leste Asiático - Coreia). Concluem que as pesquisas sobre motivação docente só terão valor prático se os seus achados forem entendidos como sendo limitados por uma série de fatores culturais, que devem ser considerados e compreendidos. 
No estudo de Klassen, Tze, et al. (2010), a eficácia coletiva correlacionou-se positivamente com a satisfação no trabalho docente, mesmo considerando a realidade de diferentes países, a saber: Canadá $(r=.42, p<.01)$; Estados Unidos $(r=.37, p<.01)$; Coreia $(r=.48, p<.01)$.

Notou-se, que a eficácia coletiva e a satisfação no trabalho são variáveis que também se podem influenciar mutuamente. Os professores que estão muito satisfeitos com a sua experiência de trabalho relatam níveis mais elevados de crenças de eficácia coletiva (Bandura, 1997, 1998; Klassen, Usher, et al., 2010). Os resultados sugerem que o investimento na melhoria das crenças de eficácia coletiva pode elevar o nível de satisfação no trabalho e, consequentemente, melhorar o desempenho do professor.

0 vértice situação socioeconômica (SES) foi o quarto elemento mais importante do multigrafo, com PageRank de 1,7, conectando-se a outros quatro vértices. Houve correlação positiva entre os elementos avaliação da prática docente e competências do ensino com o vértice autoeficácia, este, por sua vez, correlacionou-se positivamente com desempenho de alunos e eficácia coletiva.

A variável situação socioeconômica conectou-se ao vértice eficácia coletiva formando um par de vértices com três ligações, atestando que três artigos investigaram esta relação, isto indica uma conexão mais forte entre este par de vértices. Entende-se que desvantagens sociodemográficas e socioeconômicas estão associadas ao rebaixamento ou à elevação das crenças de eficácia coletiva docente. Acredita-se que diante de crenças coletivas elevadas, os efeitos negativos dos aspectos sociodemográficos são reduzidos (Parker, Hannah, \& Topping, 2006). Por isso é importante investir na elevação das crenças, uma vez que, provavelmente, é mais fácil mudar o nível das crenças de eficácia coletiva do que os aspectos sociodemográficos, pois existem implicações políticas e estruturais envolvidas nesse processo (Parker et al., 2006). É preciso considerar que, provavelmente, os professores com crenças coletivas elevadas consigam um bom desempenho no processo de ensino-aprendizagem, a despeito das desvantagens socioeconômicas das escolas (Cybulski et al., 2005).

Goddard et al. (2004) realizaram um estudo para examinar a relação entre eficácia coletiva e variáveis de contexto escolar, numa amostra de 96 escolas de Ensino Médio. A situação socioeconômica das escolas foi positivamente correlacionada com as crenças coletivas dos professores $(r=0.31)$. Ou seja, os professores tendem a ter um senso mais forte de capacidade de grupo quando instruem alunos pertencentes a classes sociais mais altas.

0 quinto vértice mais importante do multigrafo das correlações positivas foi controle externo, com PageRank de 1,6, estabelecendo conexão com três outros elementos. Houve ainda correlação positiva entre controle externo e os vértices organização do ensino e burnout. 0 vértice despersonalização foi correlacionado positivamente com controle externo.

Notou-se ainda que houve correlação positiva entre o vértice exaustão com os elementos conflito com parentes e problemas de comportamentos dos alunos. Estes foram as variáveis que estabeleceram as mais fracas conexões neste multigrafo (Figura 1), com apenas uma aresta entre os pares de vértices, indicando que apenas um artigo investigou esta relação (Skaalvik \& Skaalvik, 2007).

Variáveis - correlações negativas

Para análise das correlações negativas apresenta-se um multigrafo de relações. Na Figura 2, são demonstradas as correlações negativas entre as variáveis decorrentes dos resultados dos artigos levantados nesta revisão sistemática.

A Figura 2 representa a síntese das correlações negativas identificadas nos artigos selecionados. Destacou-se os vértices mais importantes por ordem de centralidade, a saber: (1) eficácia coletiva; (2) tempo de serviço; (3) autoeficácia. Utilizou-se como ponto de corte escores de PageRank $>1,4$. Entendese que este ponto de corte indica melhor as variáveis mais importantes dos artigos. 


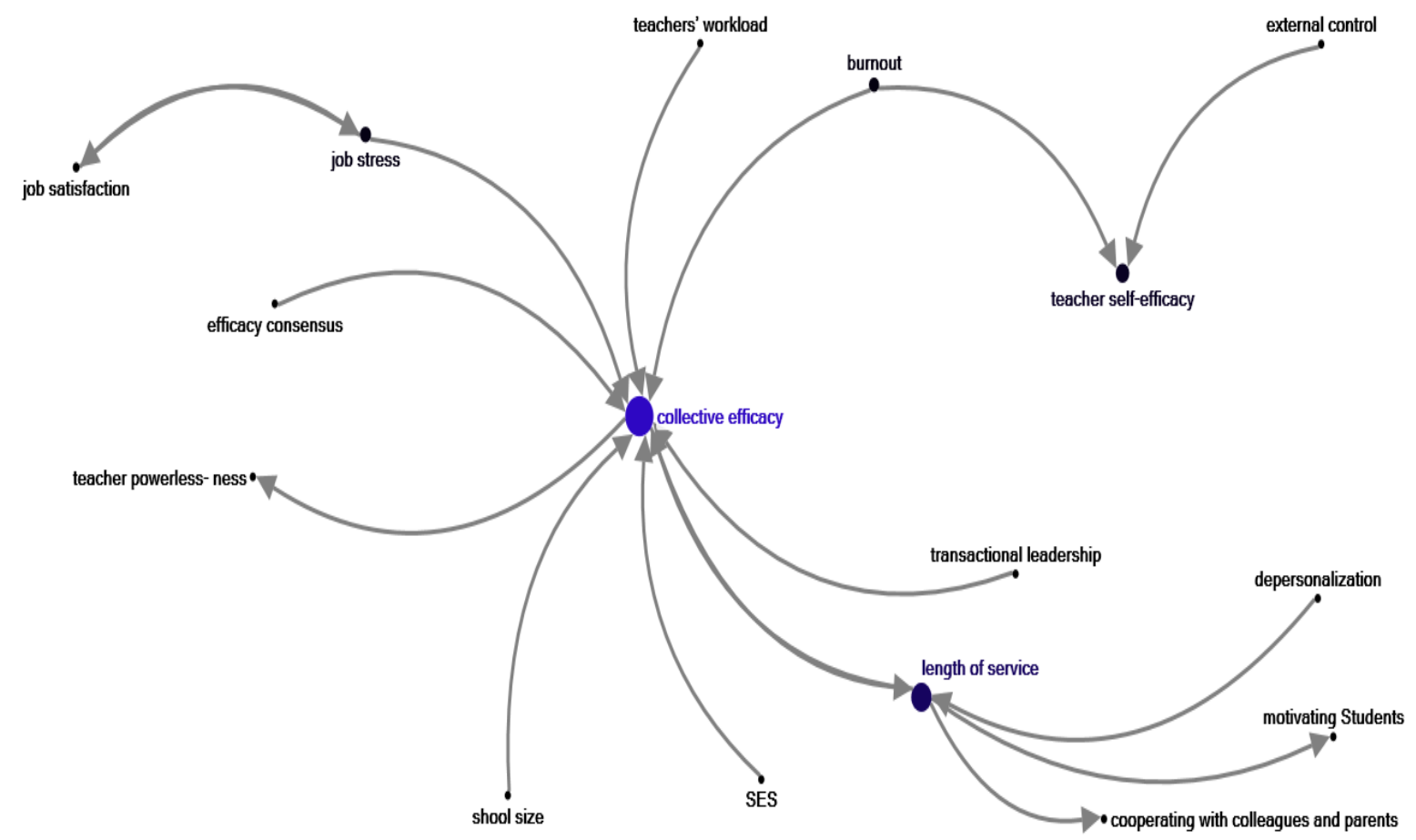

Created with NodeXL (http//hodex.codeplex.com)

Figura 2. Multigrafo - correlações negativas

A principal variável do multigrafo foi a eficácia coletiva, com nível de relevância (PageRank) 5, conectando-se a nove diferentes vértices. Houve correlação negativa entre as variáveis liderança transacional, consenso de eficácia, tamanho da escola, carga de trabalho, sentimento de impotência e situação socioeconômica, burnout, estresse no trabalho com o vértice eficácia coletiva.

Adams e Forsyth (2006) investigaram a influência das variáveis contextuais sobre as crenças de eficácia coletiva. A pesquisa realizou-se com a participação de 595 professores de 79 escolas de Ensino Fundamental e Médio, na cidade de Oklahoma, nos Estados Unidos. Sugere-se, neste caso, que escolas com elevadas condições de qualidade (boa situação socioeconômica) são predispostas a impedir a eficácia coletiva. Entende-se que algumas condições escolares e de organização institucional podem ter interferido negativamente, tais como o excesso de cobrança de superiores, ou mesmos dos pais de alunos quanto à prática docente.

Estes resultados apontam situações que podem influenciar o rebaixamento das crenças de eficácia coletiva. Quando os professores são sobrecarregados com longas cargas de trabalho e se deparam com salas de aula superlotadas, em escolas com grande número de alunos, tendem a desenvolver o sentimento de impotência, comprometendo as crenças coletivas, podendo desenvolver estresse no trabalho e o burnout.

0 segundo vértice mais importante foi tempo de serviço, com PageRank de 2,8, conectando-se a quatro outros vértices do multigrafo (Figura 2). Tempo de serviço correlacionou-se negativamente com motivação de estudantes e cooperação com colegas e familiares. Tempo de serviço foi correlacionada negativamente ao vértice despersonalização. Notou-se dupla aresta entre tempo de serviço e eficácia coletiva, indicando mais forte ligação que aponta para uma correlação negativa entre as variáveis.

Skaalvik e Skaalvik (2007), no estudo com professores de Ensino Fundamental e Médio, investigaram a relação entre tempo de serviço e eficácia coletiva. Os resultados indicaram correlação negativa entre ambas as variáveis. Percebe-se, neste estudo, que quanto mais anos de serviço o professor tem, menores são as crenças coletivas dele.

0 terceiro vértice mais importante foi autoeficácia, com PageRank de 1,4, estabelecendo conexão com dois elementos. A autoeficácia docente foi negativamente correlacionada a controle externo e burnout, isto indica que quanto mais elevadas forem as crenças de autoeficácia dos professores, menos propícios estarão a desenvolver a síndrome do burnout.

Notou-se ainda que o vértice satisfação no trabalho foi negativamente correlacionado com estresse no trabalho. Pela espessura da seta indicada na Figura 2, é possível notar que a relação entre estas variáveis é mais forte, pois formam um par de vértices com duas ligações (in-degree e out-degree). 


\section{Intensidade dos Relacionamentos entre as Principais Variáveis}

A partir da identificação das variáveis mais centrais deste dataset, encontradas através das métricas de centralidade, construiu-se uma síntese indicando a intensidade das relações entre as variáveis e as suas referências. Para tanto, analisou-se os coeficientes de correlação, entendendo que quanto mais próximo de 1 ou -1 for o coeficiente, mais forte será a correlação. Desta forma, é possível medir o nível de proximidade descobrindo se existe uma relação entre as variáveis e de que natureza ela é. Considera-se, como pré-requisito, apenas a inclusão de correlações significantes $(p<0,05)$ (Dancey \& Reidy, 2006). Assim, no Quadro 1 apresenta-se o resumo dessas referências, tomando por base as variáveis com percentual de frequência $>14 \%$, ou seja, presença em mais de quatro artigos.

Quadro 1. Intensidade das relações - variáveis mais relacionadas à eficácia coletiva docente

\begin{tabular}{|c|c|c|}
\hline Variáveis & Referências & Natureza da relação \\
\hline \multirow[t]{3}{*}{ Autoeficácia } & Parks, Solmon, e Lee, (2007) & Correlação forte \\
\hline & $\begin{array}{l}\text { Goddard, Hoy, e Hoy, (2000); Skaalvik e Skaalvik, (2007); Kurt e Duyar } \\
\text { (2012); Ware e Kitsantas, (2007) }\end{array}$ & Correlação moderada \\
\hline & $\begin{array}{l}\text { Goddard et al., (2004); Fives e Loomey (2009); Viel-Ruma, Houchins, } \\
\text { Jolivette, e Benson, (2010); Çalik, Sezgin, Kavgaci e Kilinç (2012); Kurz e } \\
\text { Knight, (2004); Lev e Koslowsky, (2009) }\end{array}$ & Correlação fraca \\
\hline \multirow{3}{*}{$\begin{array}{l}\text { Desempenho } \\
\text { alunos }\end{array}$} & Goddard, (2001) ; Goddard, (2002) & Correlação forte \\
\hline & $\begin{array}{l}\text { Goddard et al., (2000); Hoy et al., (2002); Moolenaar et al., (2012) } \\
\text { Parker, Hannah, Topping (2006); Ware e Kitsantas, (2007) }\end{array}$ & Correlação moderada \\
\hline & Cybulski et al., (2005); Goddard et al., (2004); Adams e Forsyth, (2006) & Correlação fraca \\
\hline \multirow[t]{2}{*}{ SES } & Goddard et al., (2004); Hoy et al., (2002) Cybulski et al., (2005) & Correlação forte \\
\hline & $\begin{array}{l}\text { Adams e Forsyth, (2006); McCoach e Colbert, (2010); Gibbs e Powell, } \\
(2012)\end{array}$ & Correlação moderada \\
\hline
\end{tabular}

Nota. SES - socioeconomic status (Situação socioeconômica)

Para a categorização da intensidade das relações, consideraram-se os seguintes parâmetros correlacionais: sem correlação (0); fraco $(0,1 /-0,1$ a $0,3 /-0,3)$; moderado $(0,4 /-0,4$ a $0,6 /-0,6)$; forte (0,7/-0,7 a 0,9/-0,9); perfeito (1) (Dancey \& Reidy, 2006). A análise das relações entre autoeficácia e eficácia coletiva indica a predominância de correlações moderadas e fracas. 0 desempenho de alunos foi mais moderadamente correlacionado com eficácia coletiva. A situação socioeconômica (SES) apresentou correlações de moderadas a fortes em relação à eficácia coletiva.

Estes dados indicam as correlações entre as variáveis, porém, não se pode, a partir disto, apresentar informações sobre a relação causal entre elas, pois um relacionamento correlacional não pode afirmar uma causalidade. A associação entre as variáveis não quer dizer, necessariamente, que uma cause a outra (Dancey \& Reidy, 2006).

É possível detectar, no entanto, no dataset desta revisão sistemática resultados de pesquisas que comprovam a relação causal entre essas variáveis, a saber: Skaalvik e Skaalvik (2007); Parks, Solmon e Lee, (2007); Kurt, Duyar e Çalik (2012) concluíram que a autoeficácia é preditora da eficácia coletiva (respectivamente $\beta=.64 ; \beta=.74 ; \beta=.42$ ), embora haja um consenso entre os pesquisadores de que as variáveis se influenciam mutuamente. Hoy et al. (2002), Goddard et al. (2004), Adams e Forsyth (2006) e Moolenaar et al. (2012) notaram que a eficácia coletiva é um preditor do desempenho acadêmico, indicando que quanto mais elevadas forem as crenças do grupo, melhores serão os desempenhos (respectivamente $\beta=.51 ; \beta=.24 ; \beta=.27 ; \beta=.48$ ). Percebeu-se, também, nesses estudos, que a situação socioeconômica pode prever a eficácia coletiva docente (respectivamente $\beta=.25 ; \beta=.35 ; \beta=.70 ; \beta=.59$ ). Assim, os caminhos indicados pelas correlações entre as principais variáveis são confirmados no estudo das relações causais, por meio das análises de regressão realizadas nos artigos.

\section{DISCUSSÃO}

O mapeamento dos dados coletados na presente revisão sistemática permitiu identificar as tendências empíricas dos artigos selecionados para o estudo das crenças de eficácia coletiva de professores, no que se refere à pesquisas correlacionais. 
Observou-se, considerando as correlações negativas e positivas, que algumas variáveis alcançaram destaque neste banco de dados por serem investigadas com mais frequência em conjunto com a eficácia coletiva docente e por alcançarem escores mais elevados na métrica PageRank a saber: autoeficácia; desempenho de alunos e situação socioeconômica.

A variável autoeficácia de professores totalizou 36\% dos estudos levantados, sendo a variável mais frequentemente investigada em conjunto com a eficácia coletiva docente. Os resultados indicaram que $100 \%$ dos artigos em questão encontraram correlação positiva entre os dois constructos (Britner \& Pajares, 2006; Chan, 2008; Ciani, Summers, \& Easter, 2008; Gibbs \& Powell, 2012; Kurt et al., 2012; Lev \& Koslowsky, 2009; Parks et al., 2007; Skaalvik \& Skaalvik, 2007; Viel-Ruma, Houchins, Jolivette, \& Benson, 2010; Ware \& Kitsantas, 2007; Zambo \& Zambo, 2008).

A variável desempenho dos alunos totalizou 33\% dos artigos encontrados, estando presente em 10 estudos. Os resultados indicaram que $100 \%$ dos artigos que investigaram a relação entre desempenho escolar e eficácia coletiva identificaram a ocorrência de correlação positiva (Cybulski et al., 2005; Goddard, 2002, 2006; Goddard et al., 2000; Goddard et al., 2004; Hoy et al., 2002; Kurt et al., 2012; Kurz \& Knight, 2004; Moolenaar et al., 2012; Putney \& Broughton, 2011).

A relação entre eficácia coletiva docente e situação socioeconômica (SES) da escola foi investigada em 20\% dos artigos, sendo seis estudos. Todos os resultados indicaram que as variáveis foram significativamente correlacionadas (Adams \& Forsyth, 2006; Cybulski et al., 2005; Gibbs \& Powell, 2012; Hoy et al., 2002; Kurz \& Knight, 2004; McCoach \& Colbert, 2010).

A ocorrência mais frequente dessas variáveis nos artigos selecionados sugere que os autores têm considerado estas variáveis para identificar que fatores favorecem ou prejudicam o desenvolvimento e a elevação das crenças de eficácia coletiva docente em contexto escolar. Outros constructos, no entanto, ainda são pouco investigados, porém podem representar um movimento de renovação nesta área do conhecimento, onde novas variáveis são estudadas visando ampliar a compreensão da eficácia coletiva, a saber (todos com apenas uma ocorrência na base de dados): tamanho da escola, disciplina escolar, raça, burnout, capacidade de confiança dos docentes, entre outros.

\section{CONSIDERAÇÕES FINAIS}

Por meio desta Revisão Sistemática realizou-se uma investigação quanto às variáveis de pesquisa dos estudos, utilizando técnicas de exploração e visualização com grafos. Os resultados aqui expostos favorecem a compreensão de como a eficácia coletiva docente vem sendo investigada nos últimos anos, contribuindo com informações que constituem um panorama das pesquisas nessa área nos últimos 13 anos.

Poucos estudos se dedicam a investigar a constituição das crenças de eficácia coletiva docente. Pesquisas futuras poderão examinar a relação entre variáveis contextuais, fontes de informação e crenças coletivas de eficácia. Considera-se importante, ainda, mais investigações sobre a relação entre variáveis sociodemográficas, culturais, econômicas e as crenças coletivas dos professores para explicar o impacto dessas variáveis no processo de ensino-aprendizagem.

Outro aspecto que necessita de atenção é a relação entre as crenças pessoais e as crenças coletivas de eficácia docente. Mais estudos são necessários para verificar o nível de interdependência entre estes constructos, bem como a forma como atuam na rotina escolar.

Os resultados da presente revisão sistemática indicam, ainda, que é preciso desenvolver estudos para explorar outras lacunas, tais como: análise das crenças de professores de ensino superior, o impacto da formação de professores nas crenças coletivas; mais estudos analisando as implicações do burnout nas crenças de eficácia docente de professores; mais estudos explorando o estresse, a satisfação no trabalho e as redes sociais.

A presente revisão sistemática possui algumas limitações decorrentes da escolha dos critérios de inclusão. Nesse estudo, as buscas dos artigos são realizadas apenas na base de dados do Portal de Periódicos - CAPES (Brasil) que possui grande acervo de pesquisas tanto a nível nacional, quanto internacional. Sugere-se que futuras pesquisas ampliem suas buscas utilizando outras bases de dados.

Ressalta-se que as técnicas de exploração e visualização dos dados com grafo, sendo por meio de representação geométrica ou por medidas de centralidade, foram adequadas à presente revisão sistemática, contribuindo para a identificação dos elementos mais importantes dos artigos selecionados, bem como para a compreensão do panorama da eficácia coletiva docente, favorecendo a sistematização e o rigor científico. 
Referências

Adams, C. M., \& Forsyth, P. B. (2006). Proximate sources of collective teacher efficacy. Journal of Educational Administration, 44(6), 625-642. doi:10.1108/09578230610704828

Azevedo, R. S. (2010). Sobrecarga do cuidador informal da pessoa idosa frágil: Uma revisão sistemática. UFMG. Retrieved from http://www.bibliotecadigital.ufmg.br/dspace/handle/1843/GCPA-8D9LAL

Bandura, A. (1986). Social foundations of thought and action: A social cognitive theory. Book. Prentice-Hall, Inc.

Retrieved

from http://books.google.de/books?hl=de\&lr=\&id=rcnJB7Wkr9YC\&oi=fnd\&pg=PA94\&dq=the+social+f oundations+of+thought+and+action\&ots=DYNNnRPwhK\&sig=ajTjjUIM2PPvAz2Qvjm4Wch-gEc

Bandura, A. (1989). Human agency in social cognitive theory. The American Psychologist, 44(9), 1175-84.

Bandura, A. (1993). Perceived SelfEfficacy in Cognitive Development and Functioning.pdf. Educational Psychologist, 28(2), 117-148.

Bandura, A. (1997). Self-efficacy: The exercise of control. New York: W. H. Freeman. Retrieved from http://books.google.com/books?id=eJ-PN9g_oEC\&printsec=frontcover\&dq=bandura+isbn:9780716728504\&hl=en\&ei=HAwYTbKsLpTmsQPp8c CPCg\&sa=X\&oi=book_result\&ct=result\&resnum=1\&ved=0CCMQ6AEwAA\#v=onepage\&q\&f=false

Bandura, A. (1998). Health promotion from the perspective of social cognitive theory. Psychology \& Health, 13(4), 623-649. doi:10.1080/08870449808407422

Bandura, A. (2001). Social cognitive theory: An agentic perspective. Annual Review of Psychology, 52, 126. doi:10.1146/annurev.psych.52.1.1

Bandura, A. (2008). Guide for constructing self-efficacy scales. Journal de Pharmacie de Belgique, 5(3), 307-337. Retrieved from http://www.ncbi.nlm.nih.gov/pubmed/18972865

Britner, S. L., \& Pajares, F. (2006). Sources of science self-efficacy beliefs of middle school students. Journal of Research in Science Teaching, 43(5), 485-499. doi:10.1002/tea.20131

Biruel, E., \& Pinto, R. (2011), Biblioteca: Um profissional a serviço da pesquisa. In Anais, artigo publicado em XXIV Congresso Brasileiro de Biblioteconomia, Documentação e Ciência da Informação, Maceió.

Chan, D. W. (2008). General, collective, and domain-specific teacher self-efficacy among Chinese prospective and in-service teachers in Hong Kong. Teaching and Teacher Education, 24(4), 10571069. doi:10.1016/j.tate.2007.11.010

Ciani, K. D., Summers, J. J., \& Easter, M. A. (2008). A “top-down” analysis of high school teacher motivation. Contemporary Educational Psychology, 33(4), 533-560. doi:10.1016/j.cedpsych.2007.04.002

Cordeiro, A. M., Oliveira, G. M. de, Rentería, J. M., \& Guimarães, C. A. (2007). Revisão sistemática: Uma revisão narrativa. Revista Do Colégio Brasileiro de Cirurgiões. doi:10.1590/S010069912007000600012

Cybulski, T. G., Hoy, W. K., \& Sweetland, S. R. (2005). The roles of collective efficacy of teachers and fiscal efficiency in student achievement. Journal of Educational Administration, 43(5), 439-461. doi:10.1108/09578230510615224

Dancey, C. P., \& Reidy, J. (2006). Estatística sem matemática para psicologia. Porto Alegre:Artmed.

Gibbs, S., \& Powell, B. (2012). Teacher efficacy and pupil behaviour: The structure of teachers' individual and collective beliefs and their relationship with numbers of pupils excluded from school. The British Journal of Educational Psychology, 82(Pt 4), 564-84. doi:10.1111/j.2044-8279.2011.02046.x

Goddard, R. D. (2001). Collective efficacy: A neglected construct in the study of schools and student achievement. Journal of Educational Psychology, 93(3), 467-476. doi:10.1037//00220663.93.3.467

Goddard, R. (2002). A theoretical and empirical analysis of the measurement of collective efficacy: The development of a short form. Educational and Psychological Measurement, 62(1), 97-110. doi:10.1177/0013164402062001007

Goddard, R. D., Hoy, W. K., \& Hoy, a. W. (2004). Collective Efficacy Beliefs:Theoretical Developments, Empirical Evidence, and Future Directions. Educational Researcher, 33(3), 3-13. http://doi.org/10.3102/0013189X033003003

Goddard, R. D. (2006). The influence of school social composition on teachers' collective efficacy beliefs. Educational Administration Quarterly, 42(2), 216-235. doi:10.1177/0013161X05285984

Goddard, R. D., Hoy, W. K., \& Hoy, A. W. (2000). Collective teacher efficacy: Its meaning, measure, and impact on student achievement. American Educational Research Journal, 37(2), 479-507. doi:10.3102/00028312037002479

Goddard, R. D., Logerfo, L., \& Hoy, W. K. (2004). High school accountability: The role of perceived collective efficacy. Educational Policy, 18(3), 403-425. doi:10.1177/0895904804265066 
Hoy, W. K., Sweetland, S. R., \& Smith, P. A. (2002). Toward an organizational model of achievement in high schools: The significance of collective efficacy. Educational Administration Quarterly, 38(1), 77-93. doi:10.1177/0013161X02381004

Klassen, R. M., Tze, V. M. C., Betts, S. M., \& Gordon, K. A. (2010). Teacher efficacy research 1998-2009: Signs of progress or unfulfilled promise? Educational Psychology Review, 23(1), 21-43. doi:10.1007/s10648-010-9141-8

Klassen, R. M., Usher, E. L., \& Bong, M. (2010). Teachers' collective efficacy, job satisfaction, and job stress in cross-cultural context. The Journal of Experimental Education, 78(4), 464-486. doi:10.1080/00220970903292975

Kurt, T., Duyar, I., \& Çalik, T. (2012). Are we legitimate yet?: A closer look at the casual relationship mechanisms among principal leadership, teacher self-efficacy and collective efficacy. Journal of Management Development, 31(1), 71-86. doi:10.1108/02621711211191014

Kurz, T. B., \& Knight, S. L. (2004). An exploration of the relationship among teacher efficacy, collective teacher efficacy, and goal consensus. Learning Environments Research, 7(2), 111-128. doi:10.1023/B:LERI.0000037198.37750.0

Lev, S., \& Koslowsky, M. (2009). Moderating the collective and self-efficacy relationship. Journal of Educational Administration, 47(4), 452-462. doi:10.1108/09578230910967437

Matos, I. M. D. de. (2013). Teoria dos grafos no ensino básico e secundário. Universidade de Aveiro. Retrieved from http://hdl.handle.net/10773/12083

McCoach, D. B., \& Colbert, R. D. (2010). Factors underlying the collective teacher efficacy scale and their mediating role in the effect of socioeconomic status on academic achievement at the school level. Measurement and Evaluation in Counseling and Development, 43(1), 31-47. doi:10.1177/0748175610362368

Moolenaar, N. M., Sleegers, P. J. C., \& Daly, A. J. (2012). Teaming up: Linking collaboration networks, collective efficacy, and student achievement. Teaching and Teacher Education, 28(2), 251-262. doi:10.1016/j.tate.2011.10.001

Parker, K.; Hannah, E.; Topping, J. K. (2006). Collective teacher efficacy, pupil attainment and socioeconomic status in primary school (R). Improving Schools, 9(2), 111-129. doi:10.1177/1365480206064965

Parks, M., Solmon, M., \& Lee, A. (2007). Understanding classroom teachers' perceptions of integrating physical activity: A collective efficacy perspective. Journal of Research in Childhood Education, 21(3), 316-328. doi:10.1080/02568540709594597

Pereira, Â. L. (2006). Revisão sistemática da literatura sobre produtos usados no tratamento de feridas. Universidade Federal de Goías. http://www.dominiopublico.gov.br/pesquisa/DetalheObraForm.do?select_action=\&co_obra=2548 8

Pinheiro, J. M. V. (2013). A investigação e as redes de conhecimento na European Network for Housing Research. Porto: [Edição do Autor]. Retrieved from http://repositorioaberto.up.pt/handle/10216/72175

Putney, L. G., \& Broughton, S. H. (2011). Developing collective classroom efficacy: The teacher's role as community organizer. Journal of Teacher Education, 62(1), 93-105. doi:10.1177/0022487110381760

Skaalvik, E. M., \& Skaalvik, S. (2007). Dimensions of teacher self-efficacy and relations with strain factors, perceived collective teacher efficacy, and teacher burnout. Journal of Educational Psychology, 99(3), 611-625. doi:10.1037/0022-0663.99.3.611

Szwarcfiter, J., L. (1984). Grafos e algorítmos computacionais. Rio de Janeiro: Campus.

Sousa, D. J. R. de. (2010, February 8). Caracterização de ligações entre utilizadores em redes sociais. Universidade do Porto. Retrieved from http://repositorio-aberto.up.pt/handle/10216/57672

Tschannen-Moran, M., \& Barr, M. (2004). Fostering Student Learning: The Relationship of Collective Teacher Efficacy and Student Achievement. Leadership and Policy in Schools, 3, 189-209. http://doi.org/10.1080/15700760490503706

Viel-Ruma, K., Houchins, D., Jolivette, K., \& Benson, G. (2010). Efficacy beliefs of special educators: The relationships among collective efficacy, teacher self-efficacy, and job satisfaction. Teacher Education and Special Education: The Journal of the Teacher Education Division of the Council for Exceptional Children, 33(3), 225-233. http://doi.org/10.1177/0888406409360129

Ware, H., \& Kitsantas, A. (2007). Teacher and collective efficacy beliefs as predictors of professional commitment. The Journal of Educational Research, 100(5), 303-310. http://doi.org/10.3200/JOER.100.5.303-310 
Ramos, Silva e Pontes

Zambo, D., \& Zambo, B. R. (2008). The impact of professional development in mathematics on teachers ' individual and collective efficacy: The stigma of underperforming. Teacher Education Quarterly, 35(1), 159-168.

Historial do artigo

Recebido 19/02/2015

Aceite $\quad 02 / 07 / 2015$

Publicado 12/2015

Apoio à publicação: Fundação para a Ciência e a Tecnologia (Ministério da Educação e Ciência, Portugal) - Programa FACC 
Eficácia Coletiva Docente 